\title{
Le savoir de l'Occident : sur les représentations du "Coup d'Abidjan", le 24 décembre 1999
}

\section{Michel Galy}

\section{(2) OpenEdition}

\section{Journals}

\section{Édition électronique}

URL : http://journals.openedition.org/conflits/391

DOI : $10.4000 /$ conflits.391

ISSN : $1777-5345$

Éditeur :

CCLS - Centre d'études sur les conflits lilberté et sécurité, L'Harmattan

\section{Édition imprimée}

Date de publication : 1 mars 2000

ISBN : 2-7384-9385-8

ISSN : 1157-996X

\section{Référence électronique}

Michel Galy, « Le savoir de l'Occident : sur les représentations du "Coup d'Abidjan", le 24 décembre 1999 », Cultures \& Conflits [En ligne], 37 | printemps 2000, mis en ligne le 20 mars 2006, consulté le 30 mars 2021. URL : http://journals.openedition.org/conflits/391 ; DOI : https://doi.org/10.4000/conflits. 391

Ce document a été généré automatiquement le 30 mars 2021.

Creative Commons License 


\title{
Le savoir de l'Occident : sur les représentations $\mathrm{du}$ "Coup d'Abidjan", le 24 décembre 1999
}

\author{
Michel Galy
}

1 Comment nommer l'événement ? Comment « mettre en concepts » les faits politiques, à la fois fugaces et mal délimités, hypothétiques et pourtant irréductibles dans leur singularité à l'analyse ${ }^{1}$, multipliés dans leurs gloses et représentations, pris dès leur nomination - et pire dès leur inscription ${ }^{2}$ dans un dense réseau de discours déjà hiérarchisés et constitués? A l'occasion du «Noël ivoirien » récent, on se propose d'expliciter "ce qu'analyser veut dire", dans un champ de savoir et de pouvoir spécifique, tourné à la fois vers l'ethnicité et le référent occidental. Dans la vision politiste - mais tout autant médiatique, journalistique, ou partitaire - d'un événement tel un coup d'Etat, il faudrait articuler en effet les contraintes issues d'un champ discursif du politique, déjà préconstruit, les silences, ignorances ou méconnaissances issus de l'habitus et de la praxis des observateurs ou locuteurs, qu'ils soient occidentaux ou autochtones. Comme ceux qui les expriment, tous les discours, loin d'être égaux, entrent en rapports de hiérarchisations complexes, se situent par rapport à des référants divers et parfois contradictoires; il s'agirait dès lors de mieux comprendre les procès nuancés de traductions réciproques des champs de pouvoirs en champs de savoirs: distinction par l'écart, surcroit de pouvoir dans une référence codée mais efficace au monde de l'ethnicité3. Référence déniée du politiste à la parole vécue elle-même, ce qui renvoie plus généralement aux effets d'inscription, neutralisant et réduisant un parlé créolisé qui semble à Abidjan particulièrement mouvant. Situation même du chercheur qui travaille certes, au delà de la presse et du discours des élites (champ « mondain » trop souvent, du diplomate, etc.), sur le français populaire tel qu'on le parle dans la capitale ivoirienne. L'habitus même du chercheur le rend en partie aveugle aux positions socio-linguistiques réciproques, mais sa position même dans le champ du savoir ne l'aveugle-t-elle pas dans ses conceptions théoriques mêmes - ou encore dans sa volonté la plus pure de théorisation la plus achevée, et souvent la plus décalée de la doxa? On a bien conscience de l'inculturation croissante - 
comme on dit dans l'Eglise - de la science politique, et des univers qui séparent les premiers pionniers africanistes, constitutionnalistes éminents - des recherches politistes d'un J. F. Bayart dont «la politique par le bas » s'origine justement dans la réintégration des savoirs sociologiques et surtout anthropologiques ${ }^{4}$. C'est peut-être, ailleurs comme ici, la force et le devenir du politique (comme autrefois de la philosophie ?) de synthétiser les représentations des différents mondes et de jouer des échelles des diverses sociétés plus ou moins intégrées dans l'Etat-Nation ${ }^{5}$. Mais comme on y reviendra en conclusion, l'acteur social joue en fait en permanence de ces univers de pensée et d'action. Ainsi la reconstitution du discours autochtone comporte un intérêt majeur, herméneutique: car après tout les acteurs africains, dans l'action politique ou le registre interpersonnel, sont bien les premiers à développer des stratégies de contournement, d'instrumentalisation, mais aussi de secret. Construction d'un champ à double référent $\mathrm{Y}$ a-t-il eu en Côte d'Ivoire constitution d'un champ politique autonome (au sens où l'entend Pierre Bourdieu) comme lieu, tenant du Réel et de l'Imaginaire des acteurs, dont les stratégies s'organisent par pôles, règles, stratégies - rigidifiant discours, habitus et conduites dans un ensemble structuré ? Il semble qu'il faille partir a contrario des duplications plus ou moins réussies (Constitution, jeu partitaire, élections) dans un écart fluctuant à la supposée norme occidentale, dans un retour sans cesse grandissant du refoulé ethnico-regional qui sans cesse informe et restructure le champ politique : tout comme en linguistique le parler s'inscrit par ses accentuations sur un fond dialectal, on pourrait soutenir que le discours politique « à la française " (discours comme ensemble de récits socialement constitués) qui a rang officiel dans les régimes si divers d'Afrique, est peut-être moins que l'écume des mots, redondants dans les médias, masquant l'ethnie cachée, mais plutôt la partie émergée et normative d'un continent qui resterait à décrypter. On assisterait en fait à une double articulation de discours, régie par des principes de traduction: le discours supposé à l'origine endogène, de l'ordre de l'interpersonnel, dont une longue durée de terrain permet de confirmer qu'il est alors, à l'instar d'une idéologie des origines, d'ordre ethnique dominant: ainsi premier dans les représentations de l'Autre ou les conduites $\mathrm{du}$ choix du conjoint. Le discours étatico-nationaliste, en partie déterritorialisé qui s'exprime dans la reconnaissance / négation de l'ethnie dans une volonté dissimulée d'équilibrage. Ce dernier discours s'articule à un référent d'origine occidentale, celui du politique en fait «à la française » pour Abidjan, à travers un processus complexe de duplication, de légitimation, des disciplines diverses. Ainsi la duplication des machines étatiques se doublerait-elle en permanence d'un décalage, d'une discordance structurelle qui se joue dans la mauvaise conscience - et parfois la duplicité : ni idéologie ni mimétisme. Mais écart ambigu de la représentation, issue d'un habitus luimême duel. Ce qui s'observe d'ailleurs dans le champ des institutions et des appareils d'Etat : environnement, condition féminine, décentralisation, etc. Autant de mots-clefs d'une modernité fantasmée, proposés d'un côté clef-en-main avec crédits à l'appui, tout aussitôt instrumentalisés ou plutôt réinterprétés selon la formule de G. Balandier pour les sociétés «à l'épreuve». Des transferts juridiques et institutionnels se produisent, des consultants ou concepteurs de projets portent la bonne nouvelle, des coopérants et techniciens agissent ces duplications. Mais toujours dans la différence - cet espace à la fois infime et irrattrapable où le discours de la Maîtrise se reproduit: gaps technologiques mais aussi conceptuels où sont proposées d'incessantes innovations des deux ordres pour analyser et agir. Presse, justice, appareil éducatif proposant et la norme et la sanction devant l'ignorance ou l'autochtonie apparaissent en termes 
foucaldiens comme d'incessantes disciplines. Dans ce fantasme de l'origine blanche ${ }^{6} \mathrm{du}$ politique les situations ethniques, connues partout dans leurs nuances, sont justement de l'ordre de la dénégation et agissent malgré un refoulement permanent. Ainsi, pour la propriété de la terre, on sait que le droit coutumier dans sa diversité est une des sources en France du droit foncier. Alors que le droit foncier ivoirien ne veut retenir que l'autorité de la chose publique et la législation coloniale: d'où le pourcentage minime des parcelles (de l'ordre de 1 à $2 \%$ ) légalement immatriculées. C'est que le Réel autochtone revient sans cesse. Mais dans l'ambiguïté, dans le cas cité, quand l'autorité coutumière s'estompe, que les systèmes de parenté évoluent (sous l'influence de l'individualisation et du marché, les lignages s'atomisent ; sous l'influence de l'héritage officiellement patrilinéaire et des cultures de rente désormais pérennes, les systèmes matrilinéaires se dissolvent); le foncier donne alors un inextricable embroglio entre l'étatique et le local, où seuls les acteurs dominants se retrouvent mais certes pas les paysans sans pouvoir, ou le système judiciaire lui-même. A l'inverse, projection et légitimation en retour sur la scène politique française vont de pair. A commencer par l'exemple caricatural de Félix Houphouët Boigny, acteur de la IVème République ${ }^{7}$. Chaque groupe, parti, leader a aujourd'hui ses correspondants, (af)filiations, accointances - avec tel camp ou parti français ${ }^{8}$. Pour prendre pour une fois un exemple non ivoirien le chef de l'opposition M. Abdulaye Wade vient au Sénégal de demander consécutivement, contre le parti socialiste du président Abdou Diouf et en période d'élections, l'arbitrage de l'armée sénégalaise... ou du délégué Afrique du P.S. français, assorti du président de Démocratie Libérale (et contre l'assentiment et l'information de ces derniers!). Rôles d'arbitrage, de proposition, d'incitation - si ce n'est de commandement, qu'ont longtemps tenu les ambassadeurs. A l'époque du "Vieux» (parti, syndicat et journal unique), faute de multipartisme et d'animation politique, on entendait couramment de surréalistes débats entre cadres ivoiriens, l'un tenant des "rocardiens » contre un supposé "chevènementiste" par exemple... Les capacités théâtrales de projection mais aussi de parabole et d'escapisme étaient plus sérieuses sans doute dans les commentaires publics sur le tyrannicide au Liberia, ou la fin de Ceaucescu: chaque auditeur comprenait alors la métaphore. De même, pendant la période de vacance relative du pouvoir où le vieux président était hospitalisé à Paris et M. Ouattara tenant la primature, la population n'était que peu surprise d'apprendre que le sort du pays était régi par fax, depuis la France... Les multiples séjours en France, les longues durées des missions à l'étranger donnent cette impression dans nombre de pays - ainsi Madagascar sous le président Ratsiraka actuellement - qui traduit en fait la dépendance de l'ancienne métropole. Le caractère lacunaire des pouvoirs africains non seulement devant l'international (institutions, groupes) mais aussi devant les dispositifs locaux (techniciens, confréries, féodalités, etc.) apparaît du même coup. Tout dernièrement le caractère militaire du pouvoir du Général Gueï, son grade, son putsch même (peut-être aussi son absence de soutien politique et populaire pendant sa retraite forcée des dernières années) poussent les « conseillers images " (mais aussi la référence permanente à la France) à l'identifier au pouvoir gaullien, en vue d'élections présidentielles à venir. Ici aussi double légitimation, combat occulté entre la violence et l'illégitimité du coup d'Etat et du personnage ${ }^{9}$. Chacun se positionne d'ailleurs et dans le champ politique interne et simultanément dans l'accentuation de la double référence (autochtonie et projection). C'est ainsi que tout en cultivant ses amitiés parisiennes, il parait évident que M. Ouattara a joué à fond dans l'extraversion possible de la scène politique ivoirienne: sans se prononcer sur l'obscur problème de sa nationalité 
d'origine $^{10}$, il est certain qu'un opportunisme de carrière lui a fait choisir l'origine burkinabé pour être gouverneur de la BCEAO. Ses proches, ayant exercé de hautes fonctions à l'étranger - ainsi son conseiller guinéen - ont permis à son clan de bénéficier des privatisations ${ }^{11}$. C'est surtout - et paradoxalement avec l'appui de la France - qu'après son éviction de la primature en 1993, le poste de $n^{\circ} 2$ officieux de F.M.I. lui a permis à la fois d'activer d'utiles relations aux USA et d'exercer de fortes pressions sur le gouvernement de M. Bédié. Mais l'extraversion peut rencontrer des blocages où on ne les attend pas : ainsi son union avec une femme d'affaires française, autrefois très proche de M. Félix Houphouët Boigny, a conforté cette apparence d'« ivoiro-étranger » aux yeux du petit peuple d'Abidjan. Là encore ambiguïté entre les deux référents : de la haine raciale d'un journal tel «Le Patriote»- chargé des basses œuvres de M. Bédié, employant un langage quasi frontiste («Afro-américain », « époux d'une Blanche », qui serait tantôt « juive », tantôt « catholique »... et donc l'épouse d'un "mauvais musulman»[sic]) - à l'analyse politico-culturaliste de l'opposition: «l'homme des américains » ou "du F.M.I », (comparé à l'ex-président Soglo du Bénin), on retrouve plus souvent juxtaposition qu'opposition. Comment passer de l'ethnique à l'international, ou plutôt se modeler sur les relations internationales pour produire un champ politique national à double référent ? Si une analyse de longue durée montrerait l'influence profonde des appareils idéologiques ${ }^{12}$ qui le produisent à travers l'enseignement de l'Histoire par exemple (mais aussi de la langue) ou la praxis des appareils administratifs, c'est un dispositif médiatique qui en pratique conforme l'opinion. Les média sont en effets inséparables de tout un appareil d'occidentalisation du jugement, où s'installe un habitus de distinction, de valeurs et de conduites. Parfois translation directe du système de reproduction d'Occident qui, par l'école, gomme les particularismes, dans un mimétisme étonnant : dans un village avikam des lagunes de Côte d'Ivoire où j'ai travaillé comme anthropologue sur la longue durée, l'instituteur baoulé apostrophait parfois les enfants en leur apprenant d'abord leur identité officielle (patronyme, prénom français) puis en les forçant au français: "plus de dialecte, soyez civilisés!» [1996]; comme sous la IIIème République d'ailleurs, un objet justement dit " témoin » véhicule d'ailleurs d'élève en élève la " honte » supposée d'une parole africaine, proférée dans l'enceinte de l'école. Tels les Antillais de F. Fanon ${ }^{13}$ chassant entre eux la pratique autochtone devant la norme métropolitaine, les journaux d'Abidjan se plaisent à colporter les impairs culturels des tenants du pouvoir, de l'opposition, ou de simples villageois trop africains à leur goût. Tour de passe-passe courant dans les coopérations de développement (secteur "informel »: «à réduire » dirait le dictionnaire des idées reçues franco-africaines...) mais aussi dans la parole présidentielle : Félix Houphouët Boigny dans les années 60, stigmatisera les cases de pisé ivoiriennes, sans que pour autant son programme "d'éradication des taudis " permette d'utiliser le géo-béton, technique adaptée et résistante mais jugée caractéristique d'un "développement au rabais». Et les villages en dur "baoulé » devinrent à grands frais autant de cimetières de la modernité, heureusement détournés et réinterpretés aussitôt ${ }^{14}$. Alimentation, vocabulaire, sexualité, croyances et représentations sont jaugées et jugées à cause de leurs homologues occidentales, les seules légitimes. Des pans entiers de l'économie ou des métiers traditionnels sont frappés d'illégitimité avant d'être détruits pour illégalité : petit commerce ambulant des bana-bana (colporteurs) urbains ou habitats précaires dans les quartiers spontanés appelés à être déguerpis ou détruits. Nous avons pu montrer ailleurs ${ }^{15}$ le caractère coercitif de l'appareil d'Etat, qui pris dans ce processus schizoïde de production forcée, 
accélérée, d'un champ politique national à "l'occidentale ", révèle sa violence fondatrice contre ses propres communautés. La violence symbolique de l'occidentalisation ${ }^{16}$ ne fait que précéder de peu l'application de la violence réelle, constituant une partie de la société en groupe stigmatisé - ce qui se retrouve au cœur du conflit récent au Liberia ou au Rwanda par exemple. Il n'est pas neutre que la capitale, mais aussi les grandes villes soient dénommées «blofwekro" (la ville des blancs), aujourd'hui, en Côte d'Ivoire - et "blancs » les africains occidentalisés. Sur le foncier par exemple, la transmission matrilinéaire est ouvertement condamnée, les processus transactionnels de sous seing privés, achat / vente (néocoutumiers mais écrits) ne sont pas reconnus; a contrario la politique officielle favorise du haut en bas de la chaîne hiérarchique l'étranger ou le migrant, plutôt que l'autochtone. Cette dernière attitude, qui vise surtout à casser le pouvoir des Maîtres de terre autochtones sert en fait souvent les intérêts très précis d'une ethnie (migrants baoulé de l'Ouest), ou d'un groupe social (notables urbains ou fonctionnaires jouant de ces ambiguïtés pour acquérir des propriétés ou des troupeaux). Mais surdéterminés par la propagande sur l'ivoirité, la xénophobie latente s'est appuyée sur cette contradiction pour passer à l'acte. Contre la théorie panafricaniste héritière du R.D.A., "la Côte d'Ivoire terre d'accueil » achève dans l'idéologie du sang et du sol la construction étatique d'un champ national du politique. Mais ce faisant se heurte aux antagonismes duels, omniprésents, basés sur l'opposition prototypique autochtonie vs allogénéité, où tout ivoirien est potentiellement le premier occupant (ou l'étranger) de l'Autre. Ainsi les conflits, au Nord de la Côte d'Ivoire, des Peuls éleveurs (appuyés par l'administration et les bailleurs au nom de l'importance de l'élevage et de la complémentarité écologique), ou à l'Ouest des migrants sahéliens (Burkina et Mali) contre les autochtones Krou et Bété s'étendent dangereusement jusqu'aux colons terriens baoulé exploitant dans l'Ouest ivoirien (appuyés sur la parole houphouëtiste : «la terre appartient à celui qui la travaille »). C'est ainsi qu'en novembre 1999, environ 15 à 20000 migrants ont dû quitter précipitamment l'Ouest ivoirien - plusieurs dizaines sans doute ayant été massacrés. Les projets fonciers de développement (Plan Foncier Rural - financement AFD - BM), ne voulant pas s'investir dans la réflexion géopolitique, ont préféré ignorer les renversements possibles de Maîtrise de terre: sous la pression démographique, d'autres pays pourraient être déstabilisés par la question foncière, notamment au Togo. L'Etat, les bailleurs, les techniciens peuvent étudier le symbolique et croire en «l'achat de terres par les migrants ", la réalité sociale se traduit en fait par un complexe rapport de prestations financières et symboliques, les uns et les autres partageant les valeurs quasi religieuses de l'alliance du premier occupant, « autochtone », avec les dieux de la brousse et donc sa prééminence politique devant les migrants ${ }^{17}$. Structurel, ce procès d'occultation et de partage de la violence symbolique peut s'observer plus ponctuellement dans l'événement politique : il demande à être décrypté, au fur et à mesure, selon les codes anthropologiques. Les acteurs eux-mêmes vivent et travestissent l'acte politique à l'usage des observateurs, dans une « mauvaise foi » toute sartrienne, trahissant aussi une conscience malheureuse de perte de l'ethnicité, le clivage en profondeur de personnalités résistant mal aux deux modes, mais aussi les transferts induits par le codage permanent du champ à l'usage des pouvoirs extérieurs, qui sont tout aussi déterminants. Ainsi le fameux complot politique ivoirien, dit justement du "chat noir " fait-il observer, clairement pour une fois, ces processus à l'œuvre. M. Houphouët Boigny, longtemps réputé proche du P.C.F. avant les indépendances - avait en effet gardé après son ralliement au charme discret du 
capitalisme une méthode de gouvernement par les complots qui n'était pas sans en rappeler d'autres. En juillet 1959, l'extérieur apprend l'élimination du ministre J.B. Mockey, ministre de l'intérieur limogé comme champion de la "néobourgeoisie " d'affaires. Militant de longue date du PCDI - R.D.A., Mockey était à l'origine de ces intellectuels urbains ${ }^{18}$ effectivement étrangers à la bourgeoisie terrienne des planteurs. Question de génération peut-être, quand Mockey contrôlant le parti unique, tentait d'écarter la vieille garde au profit de l'intelligentsia en gestation. Mais cette interprétation politiste, privilégiée par les médias occidentaux, aura été proposée, on le saura plus tard, à l'instigation de trois chefs d'Etat en visite à Abidjan : ces derniers (Niger, Haute Volta, Dahomey) craignant le ridicule pour l'Afrique des accusations " fétichistes », se situant uniquement par rapport au « référent ethnique » tel que nous l'avons défini. Derrière " le théâtre d'ombre du politique » - pour inverser l'expression de Bayart sur l'ethnicité, se déroulait en fait le fantastique complot du « chat noir », tel qu'il est connu encore de nos jours à Abidjan. Sous le terme impropre du fétichisme ${ }^{19}$, M. Félix Houphouët Boigny portait des accusations assez étonnantes, qui s'exprimaient dans une koinê de l'agression en double, très interethnique. On aurait trouvé dans le palais du président à Yamassoukro, une tête de chat, (avec une corne de bœuf dans sa gueule) et un "grigri » (ou talisman écrit en arabe) souhaitant la mort du président dans la valise parisienne de la femme du chef de l'Etat. Décryptage par des marabouts et des devins - guérisseurs, tribunal des différents groupes ethniques : tout un appareil néo-coutumier juge en première instance $M$. Mockey; décision ratifiée par le PCDI, publiée à travers un montage qui est la version ivoirienne des procès truqués, alors en usage à l'Est. Cette traduction polysémique de l'univers coutumier du « complot du chat noir ", utilisée en politique intérieure pour reprendre en main le parti unique, fut un peu plus tard réinterprétée dans le registre des relations extérieures: aux élections d'avril 1959, s'est fait jour un particularisme agni (ethnie fondatrice du Ghana de Kwame N'Kruma, frontalière des deux pays). Les événements d'Aboisso (le roi du Sanwi avait appelé au boycott des urnes) est présenté en 1965 par le Ministre des affaires étrangères, M. Alliali, comme un complot "N'zima » (ethnie commune à Mockey et à N'Kruma) pour démembrer la Côte d'Ivoire au détriment des baoulé. Guerres symboliques de l'après coup d'état Même aux chercheurs ou journalistes occidentaux supposés bons connaisseurs d'un pays, bien des termes et des concepts semblent doublement interdits, dans la connaissance et/ou la représentation. La médiatisation du putsch ivoirien en révèle nombre de cas, précisément dans le portrait du général président - qui ne sont pas sans conséquences politiques, en particulier électorales. L'origine ethnique et le passé personnel du général Gueï se conjuguent en effet pour une perception autochtone proche du "pouvoir cannibale» ou du pouvoir sans contrôle du Tyran, anti-modèle culturel rapporté par nombre de mythes et de légendes ivoiriennes. Ces stéréotypes interethniques fonctionnent alors dans un triple espace temps de référence: réfèrent précolonial, stéréotypes coloniaux, interrelations récentes dans le cadre de l'Etat Nation. Certains à une micro-échelle, d'autres par rapport à de grands groupes ethnico-régionaux, d'autres enfin dans la relation à des multiples étrangers ${ }^{20}$. Les ressortissants de l'ethnie Yacouba (d'où le général Gueï tire son origine, dans le Nord Ouest de la Côte d'Ivoire) sont couramment et de façon très prégnante dans la quotidienneté perçus comme des «mangeurs d'hommes »- héritage sans doute en rapport avec d'incessants et sanglants conflits segmentaires du XIXème siècle. Dans ce peuple des montagnes de l'Ouest, à cheval aujourd'hui sur la frontière de Liberia $^{21}$, certains détails ethnographiques : limage des dents, valorisation du sang de 
l'ennemi comme breuvage, dépeçage des corps des adversaires, etc., ont pu renforcer le stéréotype naissant. Les lieux : montagneux, forestiers, limitrophes ont pu constituer le signifié sémiotique d'un signifiant "sauvage» - à l'instar de "ces hommes des limites ", dans la Grèce antique chargés de tous les défauts, symétriques des «hommes civilisés ». Depuis l'époque coloniale les Dan ou Yacouba sont rétifs au « développement forcé » ${ }^{22}$, et portés à l'escapisme transfrontalier. Les ethnies du Centre, baoulé en particulier mais aussi des lagunes et de l'Ouest, les redoutent particulièrement, et les unions matrimoniales leur sont limitées. On a pu montrer la réactualisation d'une telle image du Yacouba, en Côte d'Ivoire, à travers le récent conflit libérien. Les «Scorpions Noirs » de Charles Taylor usaient et abusaient de l'attirail ethniciste: combattants terrifiants, parés de crânes comme autant de masques, de talismans "anti-balles ", décorés de scarifications et de peintures rituelles: jusqu'au pouvoir d'Etat, le "chairman " Taylor a joué de cette ambivalence des trois univers: soubassement ethnique du pouvoir, début de jeu politique national, projection (pour le Liberia) vers les Etats-Unis. Ainsi le général Gueï supporte-t-il volens nolens cet héritage ambigu : de même que certains de ses hommes qui, bariolés d'amulettes, ont débuté le premier coup d'Etat, après 40 ans de paix civile, il apparaît redoutable de par le stéréotype ethnique et par ses "exploits» antérieurs (1992) de l'assaut sauvage de la cité universitaire de Yopougon. L'image du pouvoir cannibale - autant relation à plaisanterie aujourd'hui que croyance partagée - n'est jamais très loin, fonctionnant dans les pratiques comme dans les représentations, militairement et politiquement efficace car socialement partagée. Plus intéressant encore, il jouit actuellement d'une réputation à double face de "féticheur " - «d'homme fort " diraient les lagunaires aux pouvoirs aussi mystérieux que mal définis. Contre lui, comment se positionnent ses adversaires en concurrence pour la présidence, dans toute la polysémie populaire de leur perception ethniciste? Le président déchu, Henri Konan Bédié, n'avait qu'une légende à opposer à la rumeur persistante de corruption, et à sa faible légitimité : celle du « fils spirituel », (et pour certains biologique...) de feu Félix Houphouët Boigny - ce qui paraît exclu formellement pour le dernier cas, n'emporte pas non plus la conviction pour le premier. Les "conseillers-images » eurent beau essayer de transformer le physique alourdi du président en « Eléphant d'Afrique » (métaphore osée sur le modèle des fauves asiatiques) la première bourrasque montra plutôt, selon la formule chinoise, « un tigre de papier »... Cette fragilité - voire incapacité intellectuelle pour certains avait sa correspondance institutionnelle: comme les observateurs l'avaient diagnostiqué, le PCDI était depuis longtemps une coquille vide, sans capacité de mobilisation réelle, avant même la fin du système de parti unique. Il resterait à décortiquer l'essentiel : comment au croisement de la dérive d'un président - héritier très contesté et d'une situation explosive (patiemment détissée du long labeur d'unification), des institutions militaires (gendarmerie, armée, garde présidentielle) se sont délitées en une nuit, tandis que l'appui extérieur français venait à manquer. Quant à l'opposant de toujours, l'historien Laurent Gbagbo, du Front populaire Ivoirien (FPI), son image porte la contradiction entre les stéréotypes bété et sa praxis constamment modérée. La surprésentation bété dans l'armée - problème récurrent que même Félix Houphouët Boigny a laissé tel quel - s'accorde certes à la réputation combative de ce peuple de l'Est, segmentaire et partisan déclaré "d'un chef sans pouvoir " très clastrien ${ }^{23}$. Dépourvus de la réputation sanguinaire des Dan, les Bété comprenaient des instances de régulation des conflits, des faiseurs de paix, des cultes esthétiques (bagnon) opposés à une pensée univoque de la violence. Un des dangers actuels vient 
certainement d'un retour de bâton devant la domination multiforme - du local à l'étatique - de leurs voisins baoulé : colonat agraire des baoulés en pays bété, région tenue à l'écart du développement, conflits violents autour de Gagnoa en 1972, etc. Excellent orateur - le Ministère de la parole fait partie de la panoplie du chef bété - $\mathrm{L}$. Gbagbo a eu sa part d'avanies: exil, emprisonnement, exclusion totale du pouvoir, tentative d'assassinat lors d'émeutes récentes. La clef de ce dispositif discursif n'était-il pas dans ce documentaire folklorisant, où en plein houphouetisme (fin des années 80) : un vieux sage bété fixant la caméra, assenait ses quatre vérités sur les qualités du consensus et le refus du pouvoir coercitif ? Mais les sociétés segmentaires étaient aussi dites autrefois " anarchiques » : chômeurs "déflatés » et compressés urbains, paysans appauvris par la chute brutale du café / cacao ( $-50 \%$ en un an) et la gouvernance néolibérale qui a détruit les mécanismes de stabilisation forment un cocktail explosif, plus qu'un nouveau «bloc historique ». Le culte naissant de la personnalité de Gbagbo, sa popularité d'homme courageux et intègre ne l'empêche pas de laisser ses partisans reprendre en sourdine le thème ethniciste de la non ivoirité de son rival senoufo (espérant ainsi rallier l'électoral baoulé). En effet, M. Ouattara, s'il a toutes les qualités pour bien des étrangers (formation franco-américaine, mariage mixte, néolibéralisme bien en cours à Paris et à Washington), obtient du vote en bloc "dyoula » une réputation ambiguë de leader nordiste et musulman. D'où sa difficulté à rallier les éléments baoulé nécessaires à l'arithmétique électorale, d'autant que son passé à la primature a mis en valeur un aspect répressif oublié à l'extérieur, vivace dans le pays : répression de Yopougon, plans d'austérité etc. Mais M. Gbagbo, malgré des affinités anti-baoulé avec les nordistes, devrait avoir bien du mal à se concilier aussi le côté dyoula. Chacun connaît par contre à Abidjan le secret de polichinelle de «l'alternance à l'ivoirienne » : que le futur président soit bété, dan, ou dyoula, nul doute que s'ouvrirait l'ère d'une "politique du ventre " absolument symétrique, mais inverse des années baoulé et houphouetiste. Tous les investissements, prébendes, postes juteux devraient aller à l'Ouest ou au Nord - tandis que les universitaires et les jeunes intellectuels recevraient leur part du butin national. Un management du territoire "par blocs » en quelque sorte, avec une alternance sur plusieurs décennies. Quoique les anthropologues s'interrogent eux-mêmes, dans le courant déconstructionniste et postmoderne, sur l'artificialité des frontières et enfin sur la pertinence des ethnies elles-mêmes, ce qu'on devrait retenir des politistes c'est qu'à ce niveau permanent d'identification et de cryptage des enjeux - aussi peu justifiée culturellement ou historiquement - l'identité ethnique ou plutôt l'identification aux «blocs ethnicorégionaux » est bien une des clefs du politique. Mais non la seule. Mais non dans une ahistoricité qui les fige en monades closes. Ne voit-on pas sous nos yeux éclater le «bloc baoulé »? Probabilistes, ces identités se perdent à la marge, autant que celle des classes: des anciens esclaves traditionnels avaient des positions de pouvoir et de richesse, et chaque parti a "son » Senoufo, Baoulé ou Bété - alibi ; le fils de Bédié luimême a épousé une reine de beauté dyoula... Nier en politique la question ethnique se révèle difficile: L. Gbagbo a provoqué un clash en refusant dans un premier temps de participer au gouvernement pour cause «d'overdose dyoula », en décomptant autant de partisans de Ouattara qu'il y avait des ministres nordistes; obtenant pour le FPI deux ministères supplémentaires, le leader bété trouva bon de nommer un baoulé et un sénoufo, prouvant du même coup ce qu'il prétendait nier. Au moment de conclure, l'actualité ivoirienne offre un baroque et magnifique exemple de double articulation du politique - commenté avec effarement, incompétence et ironie par les médias français. 
Après le dernier match Côte d'Ivoire / Cameroun, le régime du général Gueï ne trouva pas mieux que d'embastiller l'équipe ivoirienne des «Eléphants", comme sanction de son échec. Acte effectivement scandaleux et incongru, mais non pas gratuit. Chaque équipe connaît de longue date sa protection en double et le devin censé le protéger : mais un peu de recherche aurait montré qu'un journaliste ivoirien avait été emprisonné plus d'un an par M. Bédié pour avoir accusé ce dernier, dans une situation similaire, d'avoir porté malchance au pays. La première défaite de l'équipe du président touchait en fait à un registre symbolique du pouvoir, crypté vers l'extérieur - mais transparent pour tous, sur place. La maîtrise de la traduction Ainsi le jeu des différents registres discursifs en Côte d'Ivoire découvre une double articulation permanente qui ne prend sens que par rapport aux mondes de références: fond de l'ethnicité ne s'exprimant plus, dans le champ du politique, que très codé; utopie occidentale et française, amalgame de l'histoire coloniale et de la contemporaine duplication des appareils d'Etat. Quel que soit le nouveau leader, quelles que soient les péripéties de son accession au pouvoir, il n'exercerait pleinement la maîtrise du champ que dans une parole simultanément marquée par la double référence, produisant à la fois un effet de connivence $^{24}$ à l'aide d'une parole lourde, allusive, quasi-mythique du côté de l'africanité ; et un effet de distinction voire de prophétisme, dans l'annonce du langage de l'Autre, ce monde blanc sans cesse à advenir ${ }^{25}$. Maître donc de l'ambiguïté et de la conjugaison des contraires, le leader africain souvent incompris de l'Occident - qui n'entend que l'écho de son propre discours politique qu'il croit reconnaître - peut crypter son message plus fortement vers ses racines ethniques. Ce n'est qu'une fois " arrivé ", que le "En haut en haut " (comme on le dit à Abidjan) peut se permettre ce luxe suprême : sa relative vérité sur ses origines, sa fortune, ou son pays - tel Félix Houphouët Boigny sur son amour de l'or, son enfance de chef, ou l'ignorance des politiciens locaux. Par temps de dictature, le décryptage permanent constituent d'ailleurs les citoyens en ivoirologues avertis; inversement les médias locaux mais surtout internationaux ${ }^{26}$ (tels RFI, correspondants ou envoyés spéciaux peu avertis) se livrant à un codage permanent parfois maladroit ${ }^{27}$. A l'inverse de ce codage, la recherche d'investigation ou le simple rapport accéléré des événements produit un effet accélérateur et contagieux du mouvement - qui dans ce cas se traduit par une fièvre militaro-politique en Afrique francophone comme au Sénégal, Cameroun, Gabon, etc. Reste que cette mise en forme - ce «lissage ", comme on dit dans les institutions internationales - ce blanchiment de l'information est aussi le propre du sociologue ou du politiste. Alors que pour vouloir être prédictifs, un de leurs rôles serait justement de rapporter tels quels les discours tenus, leurs enchevêtrements ou hiérarchisations et de comprendre leurs divers référents. Certes ce filtrage, reconstruction permanente des enjeux locaux en termes de savoirs déjà constitués est aussi un phénomène occidental et peut-être le savoir du pouvoir. Mais ici l'opération semble double et redoublant l'aliénation autochtone: les régimes discursifs ne se juxtaposent pas, mais exercent entre eux hiérarchies et dominations. La traduction - en évolution permanente dans ses modèles, ses techniques notamment (informatique), ses cadres institutionnels fonctionne toujours dans l'écart permanent entre champ du politique africain et occidental, comme discipline et moyen de domination. Si certains acteurs exercent le double codage en toute connaissance et cynisme, comme méthode de pouvoir, leurs opposants sont tout aussi lucides. Ainsi L. Gbagbo passant du discours à l'appareil y voit le modèle d'un dualisme étatique : «Il y a deux Côte d'Ivoire pour les "Blancs". On fait tout pour qu'elle soit présentable. On y vote des budgets, une Constitution. On vous 
parle de répartition de tâches, mais on met des Blancs partout. Ça c'est la façade (...) mais il y a l'autre Côte d'Ivoire où règne Abdoulaye Diallo, conseiller spécial du président de la république (...) Là on nomme les ministres selon leur ethnie et non leur compétence. Les Blancs ne sont-ils pas là pour faire tourner les ministères ? Il y des gens qu'on arrête sans les juger (...). Notre président est très fort, je le reconnais. Et les Blancs tombent toujours dans le panneau ${ }^{28}$. Obliger à tenir un discours, en quelque sorte précontraint (on dirait aujourd'hui "formaté »...) dans un sens univoque : ce savoir de l'Occident. Dire dans le secret relatif, dans l'allusion permanente. Les acteurs sociaux, même sans pouvoir, peuvent maîtriser ce double savoir, et l'utiliser en situation, pour se valoriser, ou mieux se taire. Paradoxe de la doxa politique qui voudrait renfermer le référent autochtone comme archaïque ou politiquement incorrect - alors que le politique au quotidien se dit justement (ou se dissimule) dans cette alternance rapide des registres discursifs. D'où enfin l'exercice pervers de l'analyste de ces stratégies de métissage, d'acculturation qui sont autant de résistances : en quête d'enjeux plus ouverts, mais détruisant son objet en le décryptant, tout en produisant une distance supplémentaire au vécu, une dernière fois tiré vers la lumière dissolvante de la théorie politique.

\section{NOTES}

1. Deleuze G., Mille plateaux, Paris, Ed. de Minuit, 1980.

2. Bourdieu P., La distinction, Paris, Ed. de Minuit, 1979 ; Ce que parler veut dire, Paris, Fayard, 1982.

3. Goody J., La raison graphique, Trad, Paris, Ed. de Minuit, 1979.

4. Bayart J.F., L'Etat en Afrique, Paris, Fayard, 1989.

5. Dans le même mouvement que l'anthropologie s'interroge sans cesse sur ses méthodes et son objet, dont les réflexions de Dan Sperber (Le savoir des anthropologues, Paris, Hermann, 1982) peut-être un exemple pour la science politique non exempt de critiques.

6. Cf. Augé M., pour les peuples des lagunes « Les preuves blanches de l'existence de Dieu » in Théorie des pouvoirs et idéologie, Paris, Hermann,1975.

7. Et politiste à rebours - qui n'oubliait pas dans ce cas ses racines baoulé, puisqu'il a pu décrire la formation - charnière, dont il était avec $\mathrm{F}$. Mitterrand un des animateurs, comme « le lieu où les Blancs se mangent entre eux » - un des rares cas authentique d'un Persan qui ne s'ignorait pas comme tel...

8. On sait de longue date, bien avant l'affaire ELF, que pour des pays comme le Gabon, crédits et corruption font eux aussi d'étranges va-et-vient entre l'Afrique et Paris.

9. Galy M., « La Côte d'Ivoire, rejet du modèle, retour au réel », Le Monde Diplomatique, novembre 1992.

10. Problème qui existerait aussi, du côté ghanéen, pour M. Henri Konan Bédié.

11. Galy M., Le Monde Diplomatique, op. cit.

12. Voir Althusser L., «Idéologie et appareils idéologiques d'Etat » in La pensée, $n^{\circ} 151$, juin 1980. 
13. Peaux noires, masques blancs, Paris, Seuil, 1959.

14. Ainsi les placards et salle de bains transformés en silo à ignames (car le pur / impur baoulé exige impérativement les douches à l'extérieur).

15. Galy M., Les communautés contre l'Etat, Toulouse III, 1986.

16. Touré A., La civilisation quotidienne en Côte d'Ivoire. Un procès d'occidentalisation, Abidjan, Karthala, 1981.

17. Galy M., « Evaluation du PFR », Abidjan, DCGTx, 1991

18. D'après Baulin J., La politique intérieure d'Houphouët Boigny, Paris, Ed. Eurafor Press, 1982, acteur bien informé que nous suivons ici pour « l'affaire du Chat Noir ». 19. Il vaudrait mieux parler de sorcellerie : ici le terme conviendrait effectivement à l'objet-support, qui n'est d'ailleurs ainsi nommé que par transfert d'un vocabulaire de stigmatisation du paganisme, qui s'origine dans les ignorances et les mépris missionnaires.

20. Comme au Centrafrique pour Bokassa, mis en parallèle avec les représentations mythologiques du pouvoir par Bigo D., Pouvoir et obéissance en Centrafrique, Paris, Karthala, 1998.

21. Nous avons pu montrer la longue durée et l'efficacité des représentations du « corps ensauvagé » des guerriers Yacouba au combat lors du conflit libérien cf. « Libéria, Machine perverse ", in Cahiers d'Etudes Africaines, $\mathrm{n}^{\circ}$ 150-152, 1998.

22. Voir Marie A., Une Société segmentaire face à l'Etat, Thèse EHESS, 1981.

23. Clastres P., La société contre l'Etat, Paris, Ed. de Minuit, 1975.

24. Lors d'un entretien exclusif, le président Compaoré du Burkina pourtant peu porté sur les droits de l'homme, nous affirmait que ses geôles ne suffiraient pas, si on devait les remplir à chaque échec national...

25. A la limite, cette alternance des deux modes se traduit par un bilinguisme systématique - tel Mobutu apostrophant ses adversaires en lingala et tenant en français un discours plus modéré, à usage externe. Mais qui parle alors, du leader ou de la langue?

26. Car loin d'être un handicap à réformer par des stages professionnels, option de la coopération classique, la parole maladroite des apprentis journalistes permet parfois d'en apprendre beaucoup sur le pays.

27. Récemment (janvier 2000) les envoyés spéciaux du Monde et de Libération ont rapporté d'une même interview que Gueï déclarait vouloir « tricher sur Houphouët » sans comprendre que ce langage scolaire et approximatif signifiait « copier », "s'inspirer de... ».

28. Laurent Gbagbo interviewé par Julia Ficatier pour la Croix de l'Evénement, 6 mars 1990.

\section{INDEX}

Index géographique : Afrique de l'ouest, Côte d'Ivoire

Mots-clés : connaissance/ expertise, crises politiques, dictature

Index chronologique : 1999 